DOI: 10.2478/linpo-2014-0005

\title{
How Poles indicate people and objects, and what they think of certain forms of pointing gestures
}

\author{
Ewa Jarmołowicz-Nowikow
}

Institute of Linguistics Adam Mickiewicz University in Poznań, ewa@jarmolowicz.art.pl

\begin{abstract}
Ewa Jarmołowicz-Nowikow. How Poles indicate people and objects, and what they think of certain forms of pointing gestures. The Poznan Society for the Advancement of the Arts and Sciences. PL ISSN 0079-4740, ISBN 978-83-7654-384-0, pp. 85-95

The first gesture produced by children before they utter their first word has the form of an extended index finger. This gesture is often described as a prototypical pointing gesture and is regarded as a universal display of intentional communication in most explored cultures (Volterra et al. 2011; Tomasello 2007). It is interesting, however, that in many cultures, a pointing gesture realized with index finger extended is regarded as rude, especially when made by adults or by children past the age of learning to speak. The aim of this paper is to answer some questions concerning the form and usage of pointing gestures performed by native speakers of Polish. This paper is focused on potential determinants of the form of pointing gestures, on the Polish cultural norms for indicating people and objects, as well as on the perception of pointing gestures. The study is based on two experiments and a survey.
\end{abstract}

Keywords: non-verbal communication, Polish, gestures

\section{Introduction}

Pointing gestures are often regarded as the most primary and "evident" category of gestures (McNeill 1992). However, with the growing body of research devoted to them, their morphological, linguistic and sociocultural complexity (Haviland 2003) is gradually being discovered. Pointing gestures, probably present in all known cultures, are arousing more and more interest among researchers in the field of multimodal communication (Kita 2003).

Pointing gestures are fundamental in the development of communicative abilities and constitute an indispensable component of adult communication. Most of the developmental observations and experiments concerning pointing gestures focus on the preverbal stage and on the transition from the one- to two-word period. Researchers agree that the pointing gestures that emerge at an age of about 11-12 months play a vital role in speech development, and that they are fundamentally tied to this process (Butterworth 2003; Iverson \& GoldinMeadow 2005; Masataka 2003). The emergence of pointing gestures is commonly regarded as the onset of intentional communication. Some researchers understand them as very simple, early acts of communication (e.g. McNeill 1992); others claim that pointing gestures 
performed by infants possess most of the components of the pointing gestures made by adults, that is, with understanding of the intentions, attention and knowledge of the people with whom they are trying to communicate (e.g. Tomasello et al. 2007). Pointing gestures are the first as well as the last gestures to appear in the process of communication skill development. Concrete pointing gestures are made by most children before their first birthday, and they precede the utterance of the first word. The appearance of abstract pointing gestures is not as well defined in time - some researchers suggest that they are rarely used by children before they are twelve (McNeill 1992, 2005).

According to Bavelas et al. (2002) the assumption that a gesture has a single primary function is quite wrong, as a gesture can combine several functions. Analyses and observations conducted to date confirm that a pointing gesture, depending on the communicative situation, can have several functions at least; these include: 1) obvious indication of objects and events in the concrete world (Haviland 2003); 2) an imperative function as well as declarative (Butterworth 2003; Cochet \& Vauclair 2010); 3) the function of influencing the "attentional/mental states of others" (Povinelli et al. 2003); 4) the function of helping to establish a joint focus of attention between speaker and hearer (Louwerse \& Bangerter 2005).

Even though the question of the communicative function of gestures still seems to be controversial (e.g. Bavelas et al. 2007; Kendon 1994; Krauss et al. 1995; de Ruiter 2003), most researchers agree that pointing gestures are usually realized with the intention to communicate. However, the more research is done, the more often it is suggested that depending on communicative context, pointing gestures may have both communicative and speakeroriented functions (Bangerter 2004; Jarmołowicz-Nowikow \& Karpiński 2011).

\section{How to define pointing gestures}

Researchers are not unanimous in the way they define pointing gestures. Often "pointing gestures" and "deictic gestures" are used as synonyms. Defining certain categories of gestures is problematic in general, not only in the case of pointing or deictic gestures, as distinguishing separable categories of gestures is not possible (McNeill 2005). For example, the problem with defining deictic gestures is the same as with defining deictic verbal expression - it is very difficult to determine the boundaries of "deixis" (Levinson 2004). For this reason some researchers assume that most of, for example, the iconic gestures are deictic to a certain extent (Kendon 2005; McNeill 2005). It is therefore proposed to consider gestures not in categories of separate classes, but rather as a dimension (McNeill 2005) or continuum (Kendon 2005). A good solution to the terminological problem concerning indicating gestures is proposed by Kendon, according to whom pointing gestures form a subclass of the broad category of deictic gestures (Kendon 2005). The division into pointing gestures and deictic gestures is not sharp, as the whole class of indicating gestures may vary in the degree to which they are deictic - the deictic component may be more or less strong (Kendon 2005). "Gestures that are said to be pointing gestures are dominated by the deictic component almost to the exclusion of everything else. We may say of such gestures that they are specialized as pointing gestures" (Kendon 2005).

In the material studied, pointing gestures were excerpted from the whole range of gestures used by the subjects of the experiment. The experimental task was designed to elicit deictic gestures focused on indicating - that is, pointing gestures. 


\section{Aim of the study}

Little is known about the factors that influence the physical form of gestures (Gerwing $\&$ Bavelas 2004). Not much space in the literature is devoted to the problem of cultural norms for gesture realization, or the relation between the way people gesture and the way people perceive certain gesture realizations (Huth et al. 2012). Consequently, the aim of the present study is:

- to examine whether the referent is one of the potential determinants of pointing gesture form,

- to describe Polish cultural norms for pointing gesture use and respondents' opinions about certain forms of indicating,

- to verify whether there is a coherence between the way people behave (realize pointing gestures in this case) and their opinion about pointing gesture realization,

- to determine whether opinions about pointing gesture realization differ depending on age group.

It was assumed that the form of the pointing gestures realized by the Polish subjects of the experiments might differ depending on what is being indicated: a person or an object. As extended index finger is generally regarded in Poland as rude, especially when indicating people, hence it was hypothesized that Polish participants would more often use other forms of pointing gesture than index finger extended (e.g. open palm, gaze) when indicating people than when pointing at objects. The author is aware that the form of pointing gestures (especially the place of gesture realization) may be determined by other aspects of communication (Jarmołowicz-Nowikow \& Karpiński 2011; Jarmołowicz-Nowikow 2012); however this paper is mainly focused on the palm shape in a pointing gesture, and presents just one of a whole range of pointing gesture determinants.

It is predicted that comparison of recorded data and the data obtained through questionnaire forms may reveal differences between pointing gesture realization and people's stated opinions about indicating objects and people. This assumption is based on observations as well as on research showing that people's behavior may be at variance with declarations about their gestural behavior (Huth et al. 2012). It is also hypothesized that opinions about pointing gesture realization may differ between younger and older Polish respondents. It is assumed that older Poles will more often declare that pointing gestures indicating people or objects are regarded as impolite.

\section{Methodology}

To achieve the aim of the study, two experiments were conducted. Also a survey was carried out in two age groups to find out how pointing gestures are perceived by Polish people and what is their personal opinion about pointing gestures. 


\subsection{Experimental tasks}

In one of the experiments, the arrangement of the recording studio and the experimental task were designed to provoke situations in which participants would point at objects. Two participants facing each other (participant A and participant B) took part in each recording session. Each of the participants stood behind a separate desk (the desks were about three meters apart), on which were eleven identical paper figures marked with numbers. On participant A's desk there were additionally small pieces of paper with the numbers of all the figures. Participant A's task was to take a number at random, find the corresponding figure, and instruct participant B without saying the number of the figure. Participant B was asked to identify the figure in such a way that participant A could be sure that the identified figure was the right one. Both participants were told that they could communicate in any way, except that they could not use the numbers of the figures.

The apparent purpose of the other experimental task was to arrange a group of 10 people into a certain configuration and to photograph them. The real purpose was to elicit pointing gestures used for indicating people. One subject and a group of 10 students took part in the experiment. The subject and the group of students were facing each other. The subject was given a picture of ten people and was asked to position the people in the studio in the same way that the people were positioned in the picture, and then take a photograph of them. To elicit pointing gestures, the subject was asked not to address people using names and not to use any descriptions facilitating their identification (e.g. "the tall girl on the left") while putting them into position.

The subjects of both experiments were university students. The data under analysis consists of 25 sessions recorded as part of Experiment I (about 106 min.) and 12 sessions recorded as part of Experiment II (about $80 \mathrm{~min}$.). There were 1026 pointing gestures identified in the two experiments; however not all of them were analyzed. Only pointing gestures indicating people and objects were taken into consideration, and so 305 pointing gestures indicating place or direction were excluded from the analysis. All gestures were transcribed in Elan. The boundaries of all pointing gestures were marked using Kendon's model of gesture structure (Kendon 2005).

\subsection{The questionnaire survey}

The aim of the survey was to find out how respondents describe Polish cultural norms concerning pointing gesture realization, and to compare this with their personal opinion about various forms of pointing gestures made in certain communication contexts.

The survey was carried out in two age groups: a group of university students, analogous in terms of age to the group taking part in the recording sessions ( 82 respondents), and a group of graduates aged 50 or over ( 74 respondents). The reasons for selecting these two age groups were: 1 . to compare the results of the analysis from the recordings with the answers given by the younger group of respondents in the questionnaire (both the subjects in the recordings and one group of questionnaire respondents were university students); 2. to examine age groups representing two generations (children and parents) and to find out whether opinions about pointing gesture realization differ depending on age. 


\section{Forms of pointing gestures present in the recorded material}

Three main categories of pointing gestures were distinguished in the recorded material on the basis of their form:

- open palm;

- extended finger;

- gaze as pointing gesture.

Each of the categories consists of subcategories comprising recurrent specific realizations of the category's basic form, e.g. open palm.

1. Open Palm category:

- open palm [one hand],

- open palm [both hands together],

- open palm [hand partially open].

2. Extended Finger category:

- extended finger [one hand with index finger extended],

- extended finger [both hands together with index fingers extended],

- extended finger [one hand with index and middle finger extended],

- extended finger [one hand with middle finger extended].

3. Gaze as Pointing Gesture category

- gaze [gaze],

- gaze [gaze and head nod].

\section{Form of pointing gestures depending on the referent}

The form of a pointing gesture is strongly influenced by cultural conventions. Authors of intercultural studies assume that the pointing gesture probably exists in all cultures, and only its form is different (Müller 1996). The pointing gestures described in publications to date constitute a diverse set of gestures realized with hand, lips, head and gaze (Endrass et al. 2010; Enfield et al. 2007; Enfield 2001; Galhano-Rodrigues 2012; Kendon \& Versante 2003; Kita \& Essegbey 2001; Sherzer 1973; Wilkins 2003). The results obtained from analysis of the recorded material in the present work are specific to Polish experimental subjects.

The results of the analysis show a tendency among subjects to produce different forms of pointing gestures depending on the referent. In Experiment I, which elicited pointing gestures indicating paper figures, the most frequently made pointing gestures belonged to the Extended Finger category (86\%). The remaining pointing gestures (14\%) in Experiment I had a form typical of the Open Palm category. No evident examples of gaze having the function of pointing were noted in the material recorded during Experiment I. The reason for this may be the size and setting of the paper figures on both desks. It is supposed that using gaze as a pointing gesture in this particular experimental situation might not be precise enough for the other participant to identify the figure. 
In Experiment II the ratios between pointing gestures realized with extended finger and other forms of pointing gestures are different. Most pointing gestures $(66 \%)$ were realized without extended finger, that is with open palm $(34 \%)$ or gaze $(32 \%)$. About one third of all pointing gestures (34\%) indicating people in Experiment II had a form which was assigned to the Extended Finger category. Taking the norms of Polish politeness into consideration, the Extended Finger category on one hand, and the Open Palm and Gaze as Pointing Gesture categories on the other (these will be called the No Extended Finger category in the further part of this paper) may be regarded as opposing categories, since pointing with open palm or by means of gaze is not regarded as rude in Poland. ${ }^{1}$

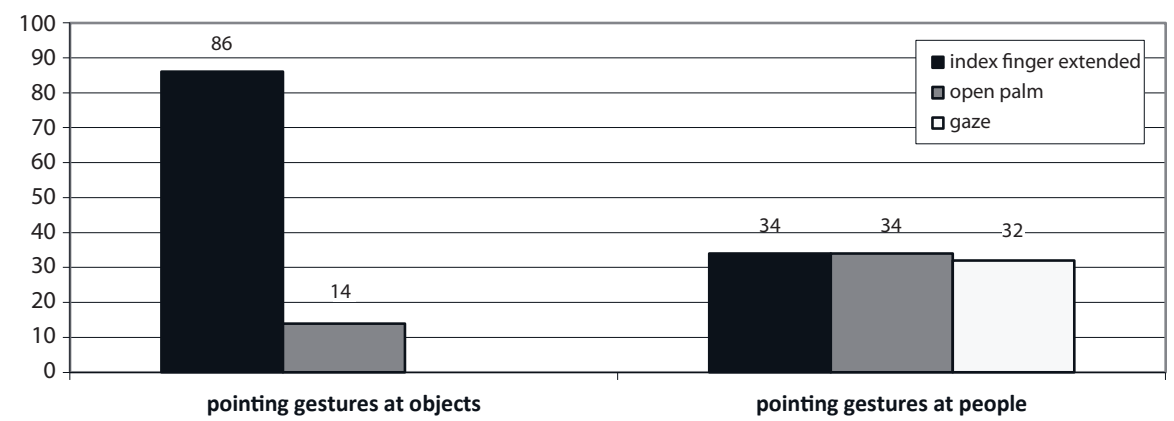

Figure 1: Form of pointing gestures used to indicate objects and people

It was also noticed that subjects were quite consistent in their "style of gesturing". This means that the predominant "style" of pointing gestures is easily distinguishable in the case of every subject. This observation applies to the recordings from both experiments. Most of the participants consistently used either gestures from the Extended Finger category, or other forms of pointing gestures without extended finger, that is gestures belonging to the categories of Open Palm and Gaze (the No Extended Finger category). The occurrence of similar proportions for gestures from the Extended Finger category and those of other categories in a single participant is very rare. The majority of participants in Experiment I (83\%) and all participants in Experiment II (100\%) had a gestural style dominated by the Extended Finger or No Extended Finger category. This means that more than $75 \%$ of the pointing gestures realized by each such participant belonged either to the Extended Finger category or to the No Extended Finger category (it does not mean that the dominant group of gestures in a single participant was always Extended Finger in Experiment I and No Extended Finger in Experiment II).

1 The results of the experiments described in this chapter are also presented in the article: JarmołowiczNowikow, Ewa. 2014. Pointing by hand; Types of reference and their influence on gestural form. In Cornelia, Müller \& Cienki, Alan \& Fricke, Ellen \& Ladewig, Silva H. \& McNeill, David \& Bressem, Jana (eds.), Body Language - Communication: An International Handbook on Multimodality in Human Interaction. (Handbooks of Linguistics and Communication Science 38.2), 1824-1833. Berlin-Boston: Mouton De Gruyter. 


\section{Survey on pointing gestures}

The results of the surveys conducted among the two age groups show that a pointing gesture, depending on its form and the context in which it is used, is perceived either as neutral or as rude. The interpretation of the gesture is also affected by the age of the people surveyed - significant differences were found for certain of the responses given by the older and younger groups.

All respondents in both age groups agreed that pointing at a person with a finger is considered rude behavior in Poland. When asked about their personal opinions on the subject, the respondents in the older group continued to state unanimously that they consider it rude behavior, while in the younger age group, $24 \%$ considered it to be a neutral behavior. These results indicate a statistically significant difference $(p<0.001)$ between the age groups as regards personal evaluation of gestures. Both analyzed groups of Poles turned out to be less severe in their evaluations of pointing at objects. No significant differences were found between the replies concerning cultural norms relating to that gesture $-66 \%$ of the younger group and $57 \%$ of the older group stated that finger pointing at objects is a neutral behavior according to Polish cultural norms. However, statistically significant differences $(p<0.001)$ between the age groups were found as regards personal evaluation of such a gesture. In this case significantly more younger respondents $(93 \%)$ than older $(57 \%)$ consider the gesture of finger pointing at objects to be neutral.

It was also asked whether other forms of indication, such as pointing at objects or people with an open palm, are considered impolite according to Polish cultural norms. In both age groups, the majority declared that pointing with an open palm, whether at objects or at people, is not regarded as an impolite behavior ( $85 \%$ of the younger group and $78 \%$ of the older group in the case of pointing at a person, and $99 \%$ of the younger group and $90 \%$ of the older group in the case of pointing at an object). In the case of pointing at people with open palm, no significant differences were found between the age groups $(p>0.05)$. A greater difference was noted in the case of interpretation of the gesture of open palm pointing at objects $(p<0.05)$ - almost all of the younger respondents regarded this type of gesture as a behavior that is perceived in Poland a neutral.

Respondents were also asked whether they believed that there had been a change in perceptions of pointing gestures in Poland. Replies to this question indicate a high level of agreement between the groups of respondents. Both among the students and among persons over 50 years of age, $77 \%$ stated that the pointing gesture has always been and continues to be perceived as rude in Poland.

Almost all of the younger respondents (99\%) stated that they remember a situation in which a family member or teacher told them off for "pointing". Almost one quarter of the older respondents do not recall such a situation. The difference $(p<0.001)$ in the response frequencies between the two groups is probably a result of the distance in time since such a situation would have occurred. Respondents were also asked whether they had ever encountered advice concerning good manners which concerned finger pointing. In this case again there was good agreement between the two groups, with approximately two-thirds of responses in the affirmative. When asked about their knowledge of cultures in which finger pointing is regarded as neutral or impolite (other than Polish), the majority of both younger and older respondents did not reply to the question. 


\section{Discussion}

The pointing gesture is the first gesture in the process of development of communication skills that is considered to be performed in an intentional manner. It is a gesture which closely precedes a child's first words. It is at the stage when a child first begins to speak, in checking whether the words are properly understood, that we encourage the child to point at their referents. Pointing, usually with a finger, is perceived as natural and neutral at this stage of language acquisition. As acquisition of language proceeds, the pointing gesture begins to be perceived as inconsistent with Polish cultural norms, and even to be suppressed. In the author's personal experience, when students are asked during classes what rules apply to the pointing gesture in Poland, they agree that it is a forbidden gesture. Some add that every child knows "not to point". When asked to consider whether the form of the gesture and the way in which it is interpreted might be context-dependent, they admit that the finger pointing gesture need not always be negatively perceived.

The chief objective of the experiments and questionnaire survey was to verify whether the form of a pointing gesture differs depending on what is being indicated, to describe the relationships between respondents' declarations concerning Polish cultural norms and their own opinions on various forms of pointing, to ascertain whether respondents' age affects the way in which pointing gestures are interpreted, and to investigate whether the manner of performance of pointing gestures is in accordance with respondents' opinions concerning their use depending on the communicative context.

Analysis of the results of the first part of the study revealed significant differences between the form of the gesture depending on the type of referent. A significant majority of the gestures serving to indicate a paper figure belonged to the Extended Finger category, while the majority of those used to point at people were performed using behaviors in the No Extended Finger category, that is, using an open palm gesture or gaze. The results of this part of the study confirmed the hypothesis that there is a dependency between the form of gesture and type of referent. In both experimental tasks, certain limitations were imposed with the aim of provoking a greater number of pointing gestures. In the first task the time for completion of the task was limited, while in the second the experimenter imposed the condition that the people being positioned must not be addressed by name or by reference to their appearance. The additional elements of the procedure helped create a corpus of recordings rich in pointing gestures. However they do not permit conclusions to be drawn concerning the frequency of use of pointing gestures, because the procedural elements introduced, as well as the task itself, in which spatial relations played a significant role, were such as to encourage the experimental subjects to perform such gestures more frequently. The recorded material nonetheless provides the possibility of analyzing the form of pointing gestures, which was the main goal of the experiments. Patterns relating to the form of pointing gestures used in the two tasks may provide a reliable basis for the drawing of more general conclusions concerning the form of pointing gestures used by Poles.

The second part of the study consisted of questionnaires, answered by two age groups. The results showed that, contrary to expectations, the behaviors of subjects in an experimental situation are in agreement with their opinions concerning the realization of specific forms of pointing. The younger group of questionnaire respondents, corresponding in age to the group taking part in the recordings, declared that pointing at objects with a finger is 
a neutral behavior in their opinion, while three-quarters of them consider finger pointing at people to be rude. The opinions prevalent among this group of respondents were reflected in the behaviors of the group of students taking part in the recording. Presumably the agreement between the manner of realizing pointing gestures and opinion regarding their use may be a result of a greater awareness of the performance of gestures of this type than in the case of other nonverbal behaviors. The gesture described by Huth et al. (2012) serving to draw attention to oneself for the purpose of beginning interaction, whose realization proved not to accord with that declared in questionnaires, is not a gesture which we attempt to control, because it is not a type of gesture which is unacceptable according to cultural norms. The pointing gesture, on the other hand, is a behavior that in Polish culture is subject to control, both on the part of the maker of the gesture, through consciously refraining from realizing it (at least in some of its forms), and on the part of observers, as when adults scold children for making such gestures.

The data obtained from the questionnaires also led to the conclusion that the greatest divergence between Polish cultural norms and personal opinions was to be found in the younger group. Some of the student respondents stated that they consider pointing at people with a finger to be a neutral behavior, although they are aware that Polish cultural norms do not accept such behaviors. Almost all of the younger group of respondents considered the gesture of finger pointing at objects to be a neutral behavior, even though some of them believe that such a gesture is regarded in Poland as impolite. Analysis of the responses of persons aged over 50 concerning finger pointing gestures at people and at objects shows that their responses concerning Polish cultural norms are in accordance with their own opinions on the subject.

Age proved to be a factor which has a significant effect on respondents' opinions. The responses given by both age groups indicate agreement among respondents as to Polish cultural norms, but differences arise in the declarations of personal opinions. As expected, fewer people in the younger group than in the older regard the gesture of finger pointing at people or at objects to be rude. It should be noted that in the group of respondents aged over 50 there were four times as many people as in the younger group who stated that pointing with the finger is an impolite behavior irrespective of what is being indicated (person or object).

There are many generally accepted opinions concerning the manner of production of selected gestures by representatives of particular cultures or nations. Most of these are a result of random observation or the promulgation of stereotypical opinions; only a few of them have been verified through research. The results described here, derived from analysis of the experimental and survey material, may - in spite of certain limitations - form a basis for the confirmation of certain rules concerning the finger pointing gesture as it is perceived in Poland.

\section{References}

Bangerter, Adrian. 2004. Using pointing and describing to achieve joint focus of attention in dialogue. Psychological Science 15. 415-419.

Bavelas, Janet \& Kenwood, Christine \& Johnson, Trudy \& Phillips, Bruce. 2002. An experimental study of when and how speakers use gestures to communicate. Gesture 2. 1-17. 
Bavelas, Janet \& Gerwing, Jennifer \& Sutton, Chantelle \& Prevost, Danielle. 2007. Gesturing on the telephone: Independent effects of dialogue and visibility. Journal of Memory and Language 5(2). 495-520.

Butterworth, George. 2003. Pointing is the Royal Road to Language for Babies, In Kita, Sotaro (ed.), Pointing: Where language culture and cognition meet, 9-33. Hillsdale, N.J.: Lawrence Erlbaum.

Cochet, Héléne, \& Vauclair, Jacques. 2010. Pointing gestures produced by toddlers from 15 to 30 months: Different functions, hand shapes and laterality patterns. Infant Behavior and Development 33. 432-442.

Endrass, Brigit \& Damian, Ionut \& Huber, Peter \& Rehm, Matthias \& Andrè, Elisabeth. 2010. Generating culturespecific gestures for virtual agents, dialogs. In Allbeck, J. et al. (eds.), IVA 2010, LNAI 6356, 329-335.

Enfield, Nick. 2001. 'Lip-pointing': A discussion of form and function with reference to data from Laos. Gesture 1(2). 185-211.

Enfield, Nick \& Kita, Sotaro \& de Ruiter, Jan Peter. 2007. Primary and secondary pragmatic functions of pointing gestures. Journal of Pragmatics 39(10). 1722-1741.

Galhano-Rodrigues, Isabel. 2012. "Vou buscar ali, ali acima!": A multimodalidade da deixis no português europeu. Linguistica: Revista de Estudos Linguísticos da Universidade do Porto 1. 129-164.

Gerwing, Jeniffer \& Bavelas, Janet. 2004. Linguistic influences on gesture's form. Gesture 4(2). 157-195.

Haviland, John. 2003. How to point in Zinacantán. In Kita, Sotaro (ed.), Pointing: Where language, culture and cognition meet, 139-170. Mahwah, N.J. \& London: Lawrence Erlbaum Associates.

Huth, Kerstin \& Loth, Sebastian \& De Ruiter, Jan Peter. 2012. Insights from the bar: A model of interaction. In Online Proceedings of Formal and Computational Approaches to Multimodal Communication, Opole, Poland, August 6-10, 2012.

Iverson, Jana \& Goldin-Meadow, Susan. 2005. Gesture Paves the Way for Language Development. American Psychological Society (16)5. 367-371.

Jarmołowicz-Nowikow, Ewa \& Karpiński, Maciej. 2011. Communicative Intentions behind Pointing Gestures in Task-oriented Dialogues. Proceedings of GESPIN, Bielefeld.

Jarmołowicz-Nowikow, Ewa. 2012. Are pointing gestures induced by communicative intention? In Esposito, Anna \& Esposito, Antonietta \& Vinciarelli, Alessandro \& Hoffmann, Rüdiger \& Müller, Vincent (eds.), Cognitive behavioural systems (Lecture notes in computer science vol. 7403), 377-389. Berlin-Heidelberg: Springer-Verlag.

Kendon, Adam. 1994. Do gestures communicate?: A review. Research on Language and Social Interaction 27. $175-200$.

Kendon, Adam \& Versante, Laura. 2003. Pointing by hand in Neapolitan. In Kita, Sotaro (ed.), Pointing: Where language culture and cognition meet, 109-137. Hillsdale, N.J.: Lawrence Erlbaum.

Kendon, Adam. 2005. Gesture. Visible action as utterance. Cambridge University Press.

Kita, Sotaro. 2003. Pointing: A Foundational Building Block of Human Communication. In Kita, Sotaro (ed.), Pointing: Where language culture and cognition meet, 1-8. Hillsdale, N.J.: Lawrence Erlbaum.

Kita, Sotaro \& Essegbey, James. 2001. Pointing left in Ghana: How a taboo on the use of the left hand influences gestural practices. Gesture 1(1). 73-95.

Krauss, Robert M. \& Dushay, Robert A. \& Chen, Yihsiu \& Rauscher, Frances. 1995. The communicative value of conversational hand gestures. Journal of Experimental Social Psychology 31. 533-552.

Levinson, Stephen C. 2004. Deixis and pragmatics. In Horn, Laurence \& Ward, Gregory (eds.), The handbook of pragmatics, 97-121. Oxford: Blackwell.

Louwerse, Max \& Bangerter, Adrian. 2005. Focusing attention with deictic gestures and linguistic expressions. Proceedings of the 27th Annual Meeting of the Cognitive Science Society.

Masataka, Nobuo. 2003. From index-finger extension to index-finger pointing: Ontogenesis of pointing in preverbal infants. In Kita, Sotaro (ed.), Pointing: Where language culture and cognition meet, 69-84). Hillsdale, N.J.: Lawrence Erlbaumaum.

McNeill, David. 1992. Hand and mind: What gestures reveal about thought. Chicago: University of Chicago Press.

McNeill, David. 2005. Gesture \& thought. Chicago: University of Chicago Press.

Müller, Cornelia. 1996. Zur Unhoflichkeit von Zeigegesten. Osnabrucker Beitrage zur Sprachtheorie 52. 196-222.

Povinelli, Daniel J. \& Bering, Jesse M. \& Giambrone, Steve. 2003. Chimpanzee 'pointing': Another error of the argument by analogy? In Kita, Sotaro (ed.), Pointing: Where language culture and cognition meet, 35-68. Hillsdale, N.J.: Lawrence Erlbaumaum. 
De Ruiter, Jan Peter. 2003. The function of hand gesture in spoken conversation. In Bickenbach, Matthias \& Klappert, Annina, \& Pompe, Hedwig (eds.), Manus Loquens: Medium der Geste - Geste der Medien, 338-347. Cologne: DuMont.

Sherzer, Joel. 1973. Verbal and non-verbal deixis: The pointed lip gesture among the San Blas Cuna. Language in Society 2(1). 117-131.

Tomasello, Michael \& Carpenter, Malinda, \& Lizskowski, Ulf. 2007. A new look at infant pointing. Child Development 78. 705-722.

Volterra, Virginia \& Caselli, Maria Cristina \& Capirci, Olga \& Pizzulo, Elena. 2005. Gesture and the emergence and development of language. In Tomasello, Michael \& Slobin, Dan (eds.), Beyond naturenurture. Essays in honor of Elizabeth Bates, 3-40. New York. Psychology Press.

Wilkins, David. 2003. Why pointing with the index finger is not a universal (in sociocultural and semiotic terms). In Kita, Sotaro (ed.), Pointing: Where language culture and cognition meet, 171-215. Hillsdale, N.J.: Lawrence Erlbaumaum. 